\title{
Multifunctional nitrogen-doped loofah sponge carbon blocking layer for
}

\section{high performance rechargeable lithium batteries}

Xingxing $\mathrm{Gu}^{\dagger, \neq}$, Chun-Jia Tong ${ }^{\S}$, Sarish Rehman ${ }^{\ddagger}$, Li-Min Liu $^{\S, *}$, Yanglong Hou $^{\ddagger *}$ (and Shanqing Zhang ${ }^{\dagger, *}$

${ }^{\dagger}$ Centre for Clean Environment and Energy, Environmental Futures Research Institute, Griffith School of Environment, Gold Coast Campus, Griffith University, QLD 4222, Australia

${ }^{\ddagger}$ Department of Materials Science and Engineering, College of Engineering, Peking University Beijing 100871, China

${ }^{\S}$ Beijing Computational Science Research Centre, Beijing 100871, China

ABSTRACT: Low cost, long life and high performance lithium batteries not only provide economically viable power source to electric vehicle and smart electricity grids, but also address the issues of the energy shortage and environmental sustainability. Herein, low cost, hierarchical porous and nitrogen doped loofah sponge carbon (N-LSC) derived from the loofah sponge has been synthesized via a simple calcining process and then applied as a multifunctional blocking layer for $\mathrm{Li}-\mathrm{S}, \mathrm{Li}-\mathrm{Se}$ and $\mathrm{Li}-\mathrm{I}_{2}$ batteries, respectively. Due to the ultra-high specific area $\left(2551.06 \mathrm{~m}^{2} \mathrm{~g}^{-1}\right)$, highly porous $\left(1.75 \mathrm{~cm}^{3} \mathrm{~g}^{-1}\right)$, highly conductive $\left(1170 \mathrm{~S} \mathrm{~m}^{-1}\right)$, and heteroatoms doping of $\mathrm{N}-\mathrm{LSC}$, the resultant $\mathrm{Li}-\mathrm{S}, \mathrm{Li}-\mathrm{Se}$ and $\mathrm{Li}-\mathrm{I}_{2}$ batteries with the N-LSC-900 membrane, deliver outstanding electrochemical performance stability in all cases, i.e., high reversible capacities of $623.6 \mathrm{~mA} \mathrm{~h} \mathrm{~g}^{-1}$ at $1675 \mathrm{~mA} \mathrm{~g}^{-1}$ after 500 cycles, $350 \mathrm{~mA} \mathrm{~h} \mathrm{~g}^{-1}$ at $1356 \mathrm{~mA} \mathrm{~g}^{-1}$ after 1000 cycles and $150 \mathrm{~mA} \mathrm{~h} \mathrm{~g}^{-1}$ at $10550 \mathrm{~mA} \mathrm{~g}^{-1}$ after 5000 cycles, respectively. The successful application to $\mathrm{Li}-\mathrm{S}, \mathrm{Li}-\mathrm{Se}$ and $\mathrm{Li}-\mathrm{I}_{2}$ batteries suggests that loofa sponge carbon could play a vital role in modern rechargeable battery industries as a universal, cost-effective, environmentally friendly and high performance blocking layer.

KEYWORDS: Li-S batteries, Li-Se batteries, $\mathrm{Li}-\mathrm{I}_{2}$ batteries, N-LSC, multifunctional blocking layer 


\section{INTRODUCTION}

The integration of energy storage units with large capacity, high rate capability, high efficiency, long cycle life, nontoxicity, and low cost is indispensable for practical implementation of the renewable energy sources at large scales ${ }^{1}$. Current advanced rechargeable batteries that adopt lithium intercalation compounds as cathode and graphite as anode still cannot meet the escalating demands in electric vehicles markets as well as the smart grid energy storage because of high cost and insufficient theoretical specific energy density $^{2-3}$. It is widely recognized that the general bottle neck of battery development lies in the limited specific capacity and energy density of cathodes of the rechargeable lithium batteries, compared with the development of anode. Thus, rechargeable batteries with high power density and energy density cathodes are crucial to electrochemical energy storage systems.

It is well known that the specific capacity $(C)$ depends on the number of electrons $(n)$ that involved in the electrochemical reaction and molecular weight $(M)$, according to the equation of $C=n * 26.8 / M\left(\mathrm{~A} \mathrm{~h} \mathrm{~g}^{-1}\right)$. Thus, the elements in the VIA group, such as sulfur and selenium, are attracting more and more attentions due to their two electrons transfer mechanism in charge/discharge processes ${ }^{1,3}$.

As the cathode in lithium batteries, element sulfur is able to present a huge theoretical specific capacity $\left(1675 \mathrm{~mA} \mathrm{~h} \mathrm{~g}^{-1}\right)$ and high energy density $\left(2600 \mathrm{~W} \mathrm{~h} \mathrm{~kg}^{-1}\right)^{3-7}$. Selenium cathode, has similar electrochemical properties as sulfur cathode because they are located in the same main group in the periodic table. Besides a high theoretical gravimetric capacity of selenium $\left(678 \mathrm{~mA} \mathrm{~h} \mathrm{~g}^{-1}\right)$, the selenium electrode also possesses a theoretical volumetric capacity (3253 $\mathrm{A} \mathrm{h} \mathrm{L} \mathrm{L}^{-1}$ ) that is comparable with sulfur (3467 $\mathrm{A} \mathrm{h} \mathrm{L}^{-1}$ ) due to the higher density in comparison with sulfur (2.5 times that of sulfur $)^{2,8-10}$. In addition, sulfur and selenium are cost-effective, abundant, and environmentally friendly ${ }^{3-5}$, which are regarded as promising next-generation rechargeable cathodes. 
Power density is also an important factor to the batteries for their potential application in EVs and HEVs. Among those high power density batteries, lithium-iodine $\left(\mathrm{Li}-\mathrm{I}_{2}\right)$ batteries are outstanding as it can possess the characteristic of rapid reaction kinetics, excellent reversibility $\left(\mathrm{I}_{3}^{-} / \mathrm{I}^{-}\right)$and superior rate performance. Furthermore, iodine cathode affords relative high gravimetric and volumetric energy densities $\left(330 \mathrm{~W} \mathrm{~h} \mathrm{~kg}^{-1}\right.$ and $650 \mathrm{~W} \mathrm{~h} \mathrm{~L}^{-1}$, respectively) ${ }^{11}$.

Interestingly, the $\mathrm{Li}-\mathrm{S}, \mathrm{Li}-\mathrm{Se}$ and $\mathrm{Li}-\mathrm{I}_{2}$ batteries are facing the same challenges in commercialization, among which the primary one is the dissolution of electroactive materials, i.e., polysulfide, polyselenides and iodides, during electrochemical cycling. To address this issue, researchers have been searching for every way to hold the electrode active materials in the electrode with various conductive carbon materials, such as porous carbon ${ }^{9,}$ 12-13, graphene $^{14-16}$, carbon nanotubes/nanofibers ${ }^{3,}{ }^{17}$, and conductive polymers ${ }^{18-19}$ since the carbonaceous materials possess large surface area and conductivity. Though significant improvement in cycling stabilities have been obtained from these strategies, current approaches are far from practical applications because of high cost in materials, complicated processes in manufacturing ${ }^{20-21}$, and more importantly limited performance in energy density due to the insufficient active material in the cathodes ${ }^{2}$.

Very recently, the concept of blocking layer (or interlayer), introduced between the cathode and the separator, is proposed to address the problem of electrode material dissolution. The interlayers need to be highly conductive, porous and strong so that it can facilitate the utilization of the active materials, boost the energy density ${ }^{22}$, extend life time by accommodating volume expansion and preventing the cathode collapse $e^{4,22-24}$. It has been reported that the dissolution and shuttling effect of the polysulfides and polyselenides are avoided by the use of the blockig layers ${ }^{2,4,22-26}$.

Carbon nanotubes ${ }^{23}$, graphene $\mathrm{e}^{2,25-26}$, carbon papers ${ }^{27-28}$, etc. have been proposed to fabricate the blocking layer, However, the synthesis of graphene, carbon nanotubes is complex and 
expensive. Low cost, high quality and large scale production of graphene and carbon nanotubes are still not available in the market. Due to the low cost, easy preparation and environmental sustainability ${ }^{22,29}$, the biochar derived from biomass, such as the poplar catkin $^{30}$, bamboo ${ }^{22}$, leaf ${ }^{31}$, eggshell membrane ${ }^{32}$, cassava ${ }^{33}$, bacterial cellulose ${ }^{29}$, etc., have been enthusiastically used to fabricated blocking layers for polysulfides/polyselenides in $\mathrm{Li}-\mathrm{S}$ and $\mathrm{Li}-\mathrm{Se}$ batteries. Though some encouraging results have been achieved along this direction, there are still a lot of rooms can be improved in terms of the electrochemical performances and the general applicability.

Loofah sponge as a kind of sustainable and renewable biomass material has been widely used as food and cleaning tools because of its soft but resilient fibers and low cost in China and Southeast Asia ${ }^{34-35}$. The loofah sponge (LS) is mainly composed of lignin and various kinds of cellulose, which commonly contain the $\mathrm{C}, \mathrm{H}, \mathrm{O}$ and $\mathrm{N}$ elements ${ }^{36}$. Herein, the loofah sponge as the precursor, is subject to a simple carbonization process at different temperatures $\left(500-900{ }^{\circ} \mathrm{C}\right)$ as shown in scheme 1 , resulting in a nitrogen-doped loofah sponge carbon $(\mathrm{N}-$ LSC) that possesses ultra-high specific surface area, ultra-large pore volume and high conductivity. The resultant carbonized materials are used to fabricate N-LSC membrane (NLSCM) that is incorporated between the cathode and separator. Resultant micro-/mesopores and intrinsic conductive macroporous networks of the N-LSC are beneficial to the storage and maintenance of the active materials, absorption of large volume of electrolyte and transportation of electrons. Moreover, the nitrogen-doping in the carbon framework provide additional immobilization for polysulfides, polyselenides, and iodides by chemical adsorption that is verified by the first-principle calculation in this work. All these favorable factors work together synergistically, leading to high initial and reversible discharge capacity, high coulombic efficiency, and excellent rate capability of the $\mathrm{Li}-\mathrm{S}, \mathrm{Li}-\mathrm{Se}$ and $\mathrm{Li}-\mathrm{I}_{2}$ cells. 

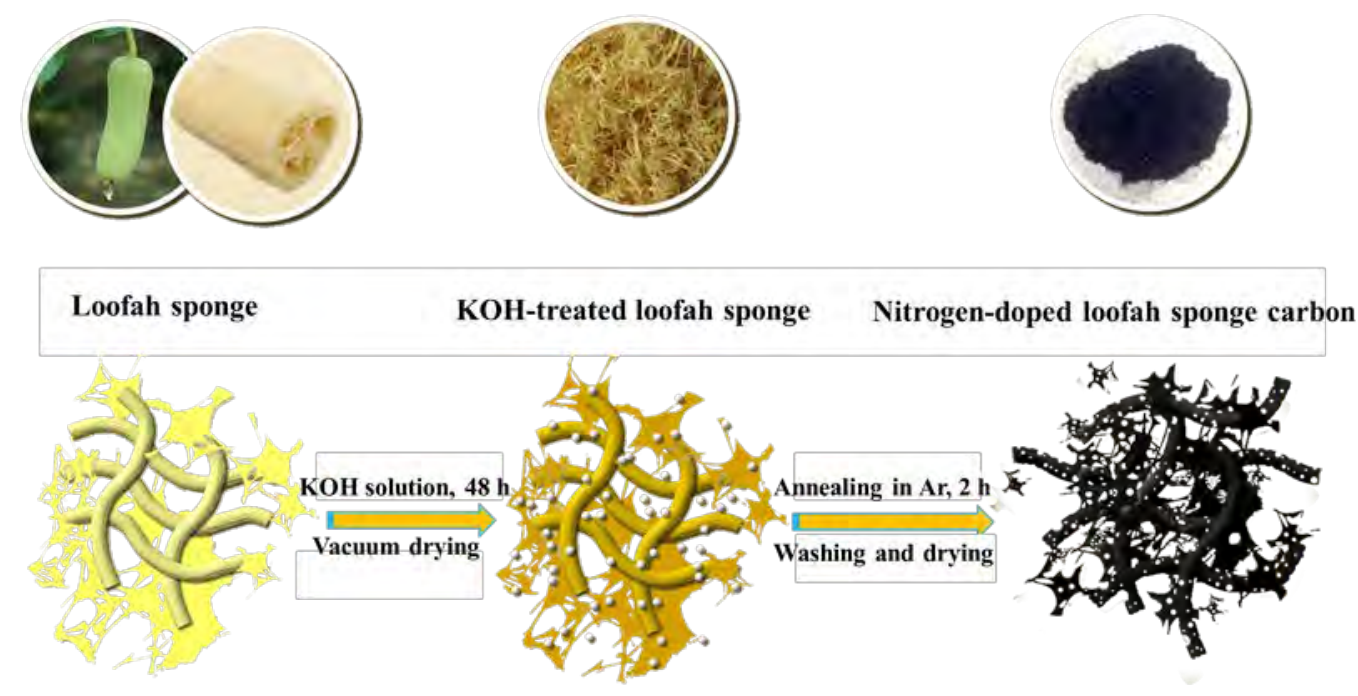

Scheme 1 The synthesis process of the nitrogen-doped loofah sponge carbon

\section{Experimental Section}

Materials Synthesis. N-LSC preparation: Firstly, the loofah sponge (LS) was immersed in $100 \mathrm{~mL}$ of $8 \mathrm{M} \mathrm{KOH}$ solution for 48 hours, filtered and vacuumed dried to get the $\mathrm{KOH}-$ treated loofah sponge. Next, obtained precursor was placed in a tube furnace, calcined at 500$900{ }^{\circ} \mathrm{C}$ under Ar atmosphere. After cooling to room temperature, the as-prepared black powder was washed with diluted $\mathrm{HCl}(1 \mathrm{M})$ solution, distilled water and ethanol in sequence to remove the residual $\mathrm{KOH}$ and organics. At last, it was dried at $60{ }^{\circ} \mathrm{C}$ in a vacuum oven. The final products were marked as N-LSC-500, N-LSC-700, N-LSC-900 according to the annealing temperature.

$N$-LSCM preparation: the N-LSC samples were pulverized and then mixed with polytetrafluoroethylene (PTFE) (analytical reagent, from Sigma-Aldrich) at weight ratio of 95:5 (N-LSC: PTFE) using ethanol (analytical reagent, from Sigma-Aldrich) as the dispersant. The pastes were rolled into films to get the nitrogen doped loofah sponge carbon membrane (N-LSCM-500, N-LSCM-700, N-LSCM-900), which the average weight, diameter and thickness are around $1.0 \mathrm{mg}, 11 \mathrm{~mm}$ and $0.1 \mathrm{~mm}$, respectively. 
$\mathrm{Li}_{2} \mathrm{~S}_{8}$ solution preparation: $0.184 \mathrm{~g} \mathrm{Li}_{2} \mathrm{~S}$ (analytical reagent, from Sigma-Aldrich) and 0.896 $\mathrm{g}$ sulfur (analytical reagent, Sigma-Aldrich) were added in $10 \mathrm{ml}$ 1,3-dioxolane $(\mathrm{DOL}) /$ dimethoxy ethane $(\mathrm{DME})(1: 1 \mathrm{v} / \mathrm{v})$ solution, stirring at $80{ }^{\circ} \mathrm{C}$ for $24 \mathrm{~h}$ to get $0.4 \mathrm{~mol}$ $\mathrm{L}^{-1} \mathrm{Li}_{2} \mathrm{~S}_{8}$ solution. And then the $\mathrm{Li}_{2} \mathrm{~S}_{8}$ solution was diluted to $2 \times 10^{-4} \mathrm{~mol} \mathrm{~L}{ }^{-1}$ for adsorption experiment.

$\mathrm{Li}_{2} \mathrm{Se}_{8}$ solution preparation: $0.186 \mathrm{~g} \mathrm{Li}_{2} \mathrm{Se}$ (Shanghai Longjin Metallic Material Co., Ltd) and $1.106 \mathrm{~g}$ selenium (analytical reagent, Sigma-Aldrich) were added in $10 \mathrm{ml} \mathrm{DOL} / \mathrm{DME}$ $(1: 1 \mathrm{v} / \mathrm{v})$ solution, stirring at $80{ }^{\circ} \mathrm{C}$ for $24 \mathrm{~h}$ to get $0.2 \mathrm{~mol} \mathrm{~L}^{-1} \mathrm{Li}_{2} \mathrm{Se}_{8}$ solution. And then the $\mathrm{Li}_{2} \mathrm{Se}_{8}$ solution was diluted to $2 \times 10^{-4} \mathrm{~mol} \mathrm{~L}^{-1}$ for adsorption experiment.

$\mathrm{LiI}_{3}$ solution preparation: $0.268 \mathrm{~g}$ LiI (analytical reagent, Sigma-Aldrich) and $0.508 \mathrm{~g} \mathrm{I}$ (analytical reagent, Shanghai Lingfeng Chemical Regent Co., Ltd) were added in $20 \mathrm{ml}$ ethanol solution to get $0.1 \mathrm{~mol} \mathrm{~L} \mathrm{LiI}_{3}$ solution. And then this $\mathrm{LiI}_{3}$ solution was diluted to $1 \times 10^{-3} \mathrm{~mol} \mathrm{~L}^{-1}$ for adsorption experiment.

Materials Characterizations. Axis Ultra imaging photoelectron spectrometer (Kratos Analytical Ltd., Manchester, UK) with a monochromatized Al Ka anode was used for X-ray photoelectron spectroscopy (XPS) measurements and the C 1 s peak at $284.8 \mathrm{eV}$ was taken as an internal standard. X-ray diffraction (XRD) patterns were obtained on a Philips X'Pert Pro (Philips Analytical, Almelo, The Netherlands) diffractometer with $\mathrm{Cu} \mathrm{K} \alpha(\lambda=1.5405 \AA)$ radiation. A Renishaw 1000 Raman imaging microscope system (Renishaw Inc, Illinois, USA) with an excitation wavelength of $514 \mathrm{~nm}$ was conducted to obtain Raman spectra. The Brunauer-Emmett-Teller (BET) method using nitrogen adsorption and desorption isotherms was performed on an ASAP 2020 system (Micromeritics, USA). Quench solid density functional theory (QSDFT) method was used to calculate the pore size distribution and pore volume from the adsorption branch of the $\mathrm{N}_{2}$ adsorption/desorption isotherms. The morphologies and structures of the obtained samples were characterized through scanning 
electron microscope HITACHI S-4800 (Japan), and a FEI Tecnai F30 (USA) transmission electron microscopy.

Electrochemical measurements. The electrode slurry was mixed the electrode materials (i.e., pure sulfur/selenium) with carbon black and polytetrafluoroethylene (PTFE) in a weight ratio of 70: 20: 10 with ethanol as the dispersant, while the ratio for $I_{2}$ is 50: 40: 10. The resultant pastes were compressed on the $\mathrm{Al}$ foil, and then cut into discs with a diameter of 8.0 $\mathrm{mm}$ and an area of $0.5 \mathrm{~cm}^{2}$. After vacuum drying at $60{ }^{\circ} \mathrm{C}$ for 12 hours, the average weights of the wafers are $1.5 \mathrm{mg}$ for $\mathrm{I}_{2}, 1.8 \mathrm{mg}$ for $\mathrm{S}, 1.8 \mathrm{mg}$ for Se respectively. The half-cells were assembled in a glove box (M-Braun, Germany) under high pure Ar atmosphere. These halfcells consists of lithium anode, Celgard 2300 separator (14 mm diameter), $1 \mathrm{M}$ lithium bis(trifluoromethanesulfonyl)imide (LiTFSI) in DOL/DME (1:1 v/v) containing $0.1 \mathrm{M} \mathrm{LiNO}_{3}$ as the electrolyte for $\mathrm{Li}-\mathrm{S} / \mathrm{Se}$ batteries and $1 \mathrm{M}$ LiTFSI with $1 \mathrm{M} \mathrm{LiPF}_{6}$ in ethylene carbonate (EC)/dimethyl carborbonate (DMC)/ethyl methyl carbonate $\left(\right.$ EMC) $(1: 1: 1 \mathrm{v} / \mathrm{v} / \mathrm{v})$ for $\mathrm{Li}-\mathrm{I}_{2}$ batteries. The LAND CT-2001A instrument (Wuhan, China) was employed to investigate the cycling performances of the half-cells with a potential window between $1.5-3.0 \mathrm{~V}$ for $\mathrm{Li}-\mathrm{S} / \mathrm{Se}$ cells and $2.0-3.8 \mathrm{~V}$ for $\mathrm{Li}-\mathrm{I}_{2}$ cells respectively, at room temperature. While the cyclic voltammetry (CV) curves was obtained from the CHI $760 \mathrm{E}$ electrochemical workstation (CHI Instrument, Shanghai, China). The electrochemical impedance spectroscopy (EIS) was also recorded using the $\mathrm{CHI}$ instrument by setting the frequency range from 100 $\mathrm{kHz}$ to $10 \mathrm{mHz}$. In addition, electrical conductivity of the obtained N-LSCM was conducted by a four-probe method on Resistivity Measurements System (RTS-8, China).

Computational Details. The computations were performed using the Vienna $\mathrm{Ab}$ Initio Simulation Package (VASP) ${ }^{37-38}$ under the framework of density functional theory (DFT). The projector augmented wave (PAW) pseudopotential was adopted and the generalized gradient approximation (GGA) with the PBE functional ${ }^{39}$ was used to treat the exchangecorrelation interaction between electrons. Particularly, the DFT-D3 method ${ }^{40-41}$ was employed 
to improve the description of long-range van der Waals (vdW) interaction. The cutoff energy of the projector augmented plane-wave basis set is $500 \mathrm{eV}$ to ensure an accuracy of the energy of $1 \mathrm{meV}$ per atom. The full geometry optimizations are carried out with the convergence thresholds of $10^{-5} \mathrm{eV}$ and $1 \times 10^{-2} \mathrm{eV} / \AA$ for total energy and ionic force, respectively. The kpoint sampling uses the Monkhorst-Pack scheme on a $3 \times 3 \times 1$ mesh.

\section{RESULTS AND DISCUSSION}

The pristine loofah sponge with a large amount of the small and thin sheets crosslinking with the ultra-long fibres is shown in Figure S1. After the calcination process at high temperatures in the presence of $\mathrm{KOH}$, the loofah fibres and the sheets have converted into the porous loofah carbon as shown in Figure 1a-d. The porous structure of N-LSC-900 (Figure 1c) becomes more obvious compared with the N-LSC-700 (Figure 1b) and N-LSC-500 (Figure 1a). That is because the $\mathrm{KOH}$ corrodes the carbon framework more intensively at higher temperature, creating more and larger micorpores ${ }^{42}$. Moreover, the SEM image of Figure 1d and TEM image of Figure 1e indicate that the macropores and mesopores range from several nanometers to few tens of nanometers, respectively. HRTEM image in Figure 1f, exhibits the micropores of N-LSC-900 sample are less than $2 \mathrm{~nm}$. This suggests that the N-LSC-900 sample has hierarchical pores, i.e., macro-, meso- and micropores. 


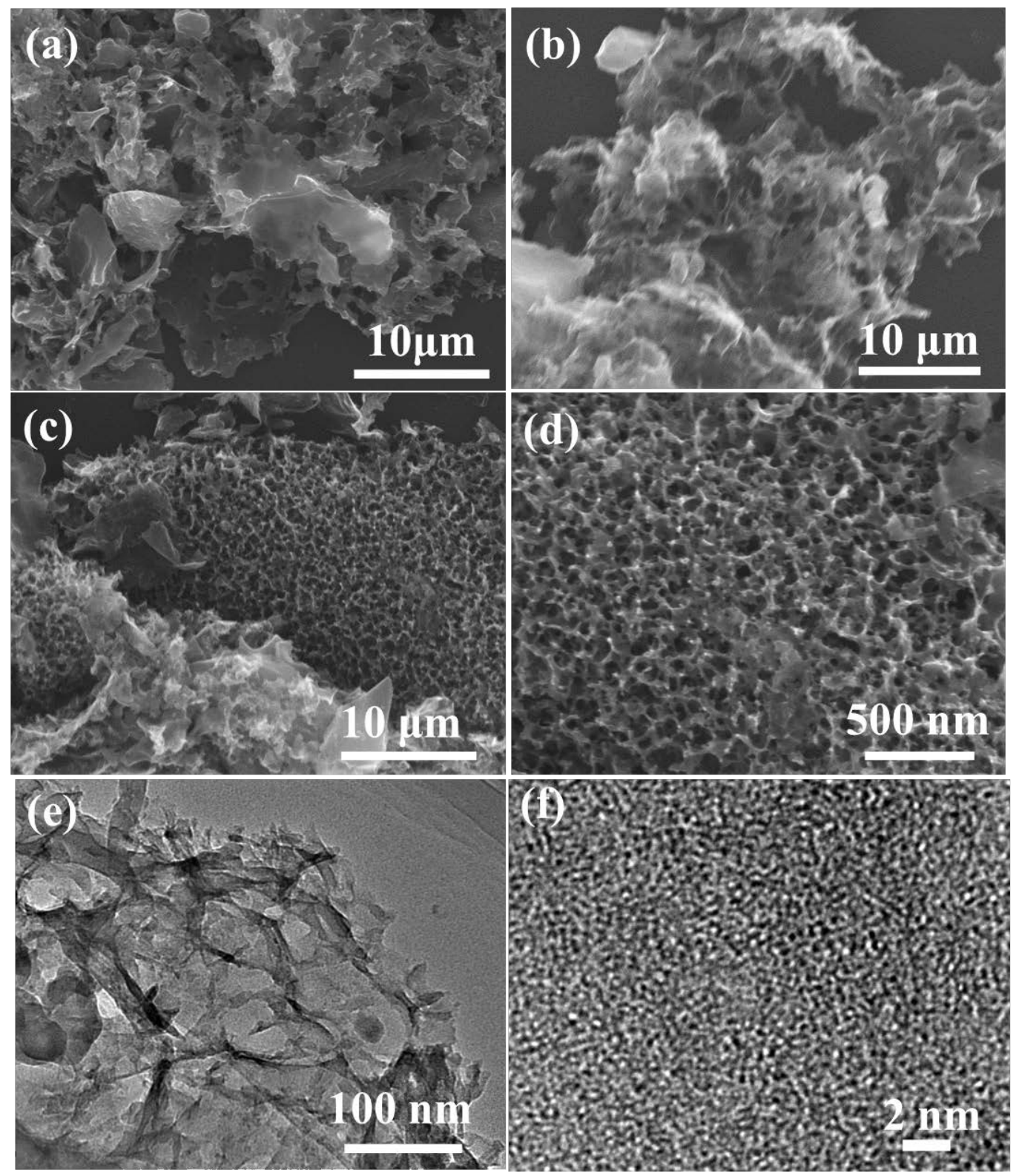

Figure 1 SEM images of (a) N-LSC-500, (b) N-LSC-700, (c-d) N-LSC-900; TEM images of (e) N-LSC-900 and HRTEM image of (f) N-LSC-900.

In order to further affirm the hierarchical pores of N-LSC, the $\mathrm{N}_{2}$ adsorption/desorption characterization has been conducted. As expected, all the N-LSC samples show the combination of type I and type IV isotherms (Figure S2a-c), indicating the typical characteristics of the micropores and mesopores ${ }^{43}$. And the pore size distributions (Figure S2d-f) also prove the abundant micropres and mesopores of the N-LSC samples. Meanwhile it can be observed the pore sizes increase with the temperature increase as shown in the Figure S2d-f, which agrees well with the SEM and TEM observation. Because of such hierarchical 
pores, the N-LSC illustrates ultra-high specific surface area and the pore volume as shown in Table S1. Particularly the N-LSC-900 owns a specific surface area of $2551.06 \mathrm{~m}^{2} \mathrm{~g}^{-1}$ and pore volume of $1.75 \mathrm{~cm}^{3} \mathrm{~g}^{-1}$. It is expected that the hierarchical pores can facilitate the electrolyte infiltration, and inhibit the polysulfides, polyselenides, and iodides shuttling ${ }^{4,8-9,12-13,22}$. In particular, the high specific surface area provides more active sites during the charge/discharge processes ${ }^{22}$, and the high pore volume accommodates more active materials. XPS was employed to investigate chemical composition at the N-LSC surface. As shown in Figure S1c and Table S3, the pristine LS contains C, O, N, which are common elements in plant tissue 22,44 . After annealing, the N-LSC remain the same types of elements. But the content percentage has changed (see Figure 2 and Table S1). The percentage of the C increases, while the $\mathrm{O}$ and $\mathrm{N}$ percentage contents decrease with the increase of the annealed temperature. That is due to the decomposition of $\mathrm{C}=\mathrm{O}$ and $\mathrm{C}-\mathrm{N}$ bond in the inert atmosphere during the annealing process ${ }^{22}$. However, even though the annealing temperature increases to $900{ }^{\circ} \mathrm{C}$, the LSC-900 still contain $1.89 \mathrm{wt} \% \mathrm{~N}$. The corresponding high-resolution $\mathrm{N} 1 \mathrm{~s}$ spectra (Figure 2b-d) illustrate the availability of three kinds of N, i.e., graphitic N $(401.2 \mathrm{eV})$, pyrrolic $\mathrm{N}(400.1 \mathrm{eV})$ and pyridinic $\mathrm{N}(398.4 \mathrm{eV})$, in all the LSC samples ${ }^{4}$. The heteroatoms doping as such in carbon framework could effectively enhance the conductivity of the materials and thus enhance the active materials utilization percentage of the cathode ${ }^{4}$. In addition, our previous study and other works in the literatures suggest that the doped-nitrogen atoms are beneficial to the adsorption of the polysulfides and polyseneides, which contributes to further improve the cycling stability of the resultant batteries ${ }^{4,45-46}$. 

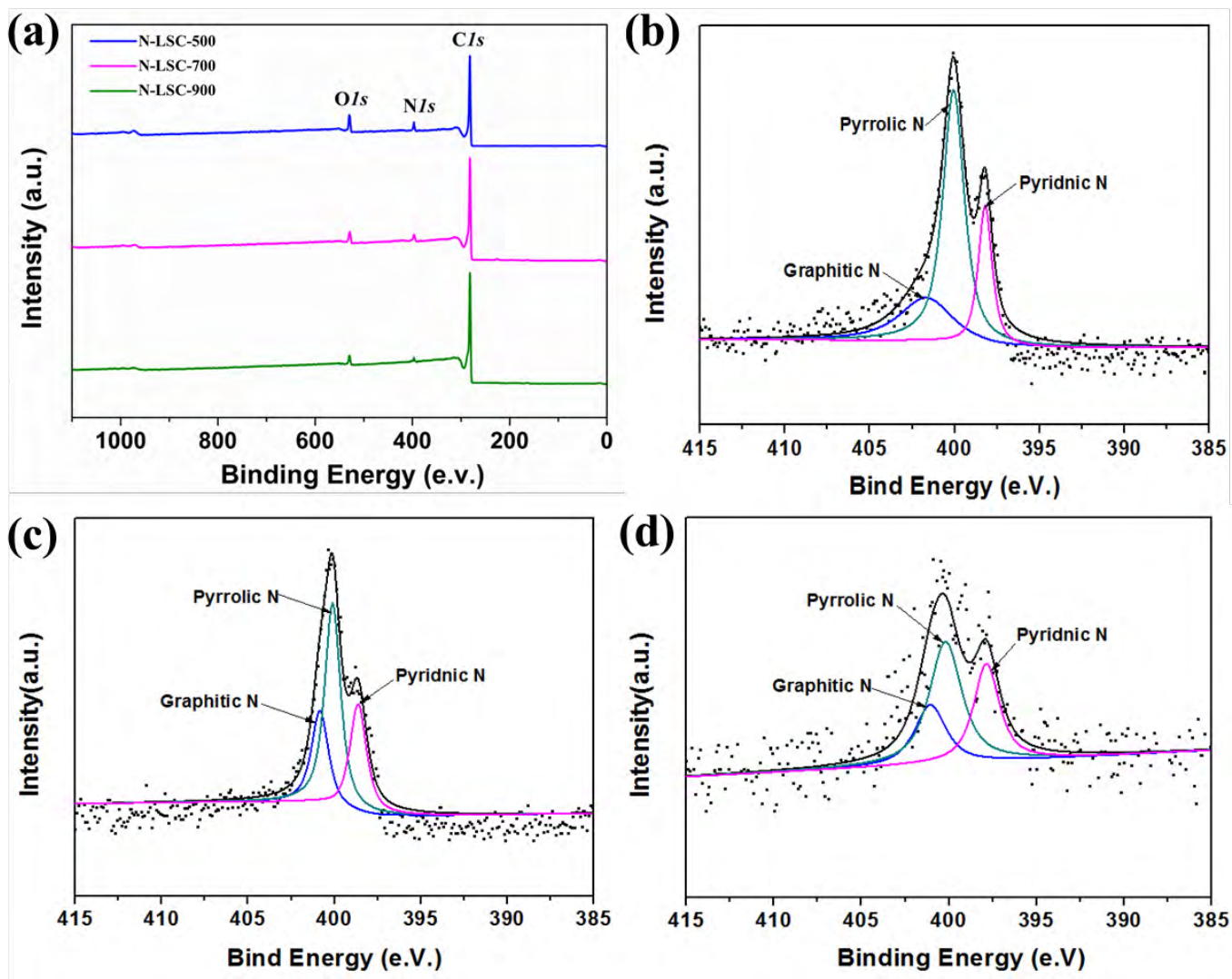

Figure 2 (a) XPS survey spectra of the LSC samples and high resolution N1s XPS spectra of (b) N-LSC-500, (c) N-LSC-700 and (d) N-LSC-900.

The crystallographic structures of the N-LSC samples were analysed by XRD. As shown in Figure S3a, two broad diffraction peaks are correspondent to the (002) and (100) facets of the hexagonal carbon, implying the weak graphitization of these samples. But with the temperature increasing, the (002) peak of the N-LSC-900 becomes sharper compared to the N-LSC-700 and N-LSC-500, suggesting highest graphitization of N-LSC-900. Further the Raman spectra in Figure S3b also confirm these results. In a Raman spectra for carbon materials, the D band corresponds to disordered carbon or defective graphitic structures ${ }^{43,47}$; while the $G$ band is a characteristic feature of the graphitic layers and corresponds to the tangential vibration of the carbon atoms ${ }^{43,47}$. All the N-LSC samples exhibit the D and G bands at ca. 1350 and $1575 \mathrm{~cm}^{-1}$ in Figure S3b. And the integral intensity ratio of D band to G band of these three samples decrease with the increasing carbonization temperature, which means higher graphitization and better conductivity ${ }^{48}$, in line with the XRD results. In 
addition, the D-band becomes broader and stronger due to the concentration of heteroatoms increases resulting more defects ${ }^{47}$. Thus the D-band in N-LSC-500 and N-LSC-700 is broader compared to that of N-LSC-900, because of the higher $\mathrm{N}$ atoms doping in the carbon framework. Both the Raman and XRD results support the conclusion that a high concentration of doped $\mathrm{N}$ heteroatoms causes increased defects in the carbon structure, resulting in a broader D-band and (002) XRD peaks ${ }^{47}$.

The four-point probe method was used to investigate the conductivity of the as-prepared NLSCM samples. Interestingly, the N-LSCM samples demonstrate excellent conductivity. Among them the N-LSCM-900 sample exhibits the conductivity as high as $1170 \mathrm{~S} \mathrm{~m}^{-1}$; while the N-LSCM-700 and N-LSCM-500 are $175 \mathrm{~S} \mathrm{~m}^{-1}$ and $1.1 \mathrm{~S} \mathrm{~m}^{-1}$, respectively. Compared with other carbon materials as shown in the Table S3, the conductivity of N-LSC-900 is far higher than other porous carbon, biochar, and activated carbon and even comparable with the graphene. The high conductivity could be attributed to partial graphitization at high temperature and the nitrogen doping.

The as-prepared N-LSCMs were firstly applied as blocking layers for the Li-S batteries. The CV curves of the Li-S batteries without the N-LSCM and with N-LSCM-500, N-LSCM700, N-LSCM-900 are shown in Figure 3a-d. It can be observed the batteries with the NLSCM interlayer illustrate higher CV peak currents, which is possible due to the higher conductivity after inserting the N-LSCM. And the batteries with the N-LSCM interlayer show better consistency of peak position compared to the batteries without N-LSCM interlayer, suggesting the polarization of the cathode has efficiently impeded after inserting N-LSCM interlayer. Particularly, the batteries with N-LSCM-900 display the weakest polarization. In addition, the batteries with N-LSCM illustrate two reduction peaks at ca. $2.33 \mathrm{~V}$ and $2.03 \mathrm{~V}$ during the cathodic scan, corresponding to the reduction of the elemental sulfur to the higher order lithium polysulfides $\left(\operatorname{Li}_{2} \mathrm{~S}_{x}, 4 \leqslant x \leqslant 8\right)$, and the reduction of the higher order lithium polysulfides to the lower order lithium polysulfides, even to the insoluble $\mathrm{Li}_{2} \mathrm{~S}_{2}$ and $\mathrm{Li}_{2} \mathrm{~S}$, 
respectively ${ }^{4,49-50}$. In the oxidation process, only one oxidation peak located at ca. $2.35-2.45$ $\mathrm{V}$ is observed in Figure $3 \mathrm{~b}-\mathrm{d}$, which is parallel with the transformation of insoluble polysulfides into the soluble polysulfides ${ }^{4,49-50}$. And both the reduction and oxidation peaks changes sharply along with the N-LSCM variation. As expectedly, the batteries with the NLSCM-900 has the sharpest oxidation peak shape and the most identical repeatability compared to other cells, indicating the best cycling stability of the sulfur electrode with the NLSCM-900 interlayer ${ }^{4}$.

Figure $3 \mathrm{e}$ illustrates the initial galvanostatic charge/discharge behaviors of the Li-S batteries with and without N-LSCM at the current density of $1675 \mathrm{~mA} \mathrm{~g}^{-1}$. Two obvious plateaus can be observed in the cells with N-LSCM interlayer, in accordance with the reduction of the elemental sulfur to the high order polysulfides at around $2.3 \mathrm{~V}$ and to the low order $\mathrm{Li}_{2} \mathrm{~S}_{2} / \mathrm{Li}_{2} \mathrm{~S}$ at approximately $2.1 \mathrm{~V}^{4,25,49-50}$, respectively. In particular, the batteries with the N-LSCM900 interlayer possess the widest and the most

stable plateaus with an extremely low polarization of $148.8 \mathrm{mV}$ (vs. $252.5 \mathrm{mV}$ for the batteries without the N-LSCM interlayer) at $1675 \mathrm{~mA} \mathrm{~g}^{-1}$, suggesting a more kinetically efficient reaction process with a smaller barrier ${ }^{4,25,51-52}$. What's more, the plateau is not obvious in the cell without the N-LSCM interlayer, indicating the high polarization and slow redox reaction kinetics with the inferior reversibility ${ }^{4,25}$. Because of the superior electrical conductivity, highly porous structure of the N-LSC, can the cells with N-LSCM illustrate better electrochemical performance. 
(a)

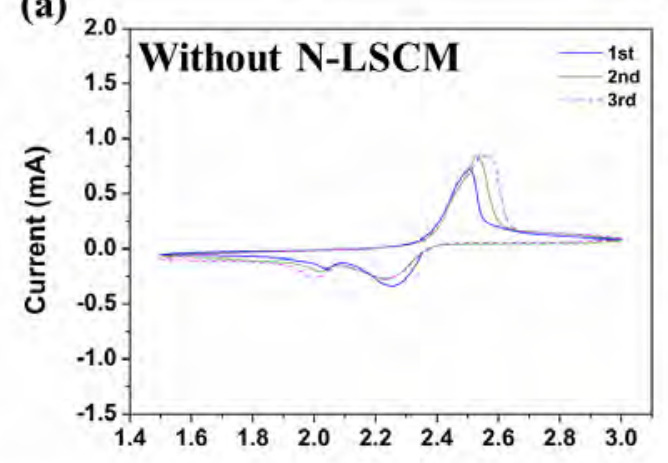

(c)

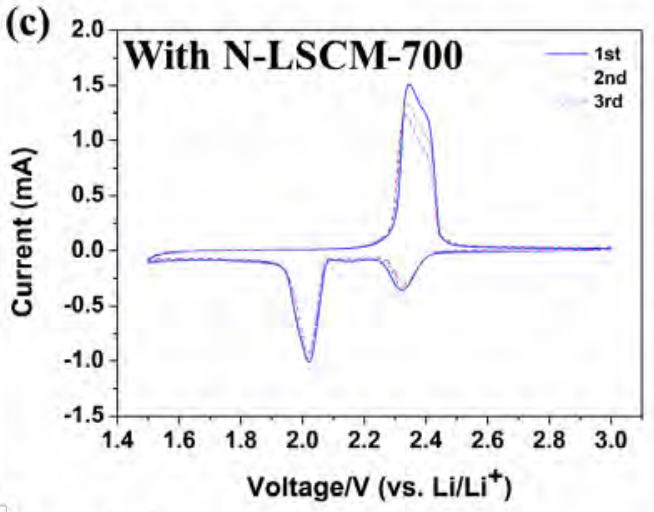

(b)

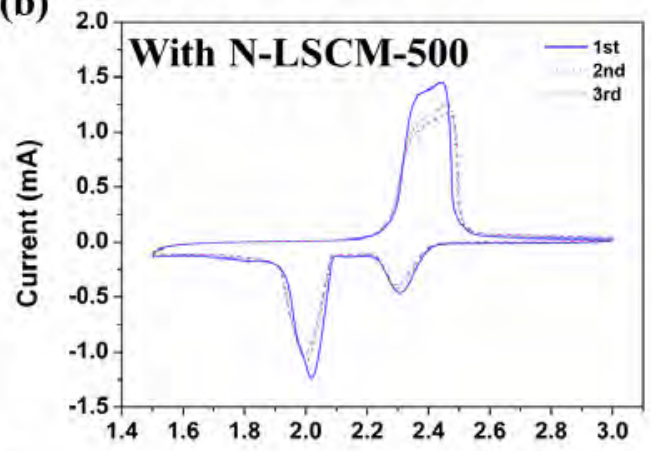

(d)

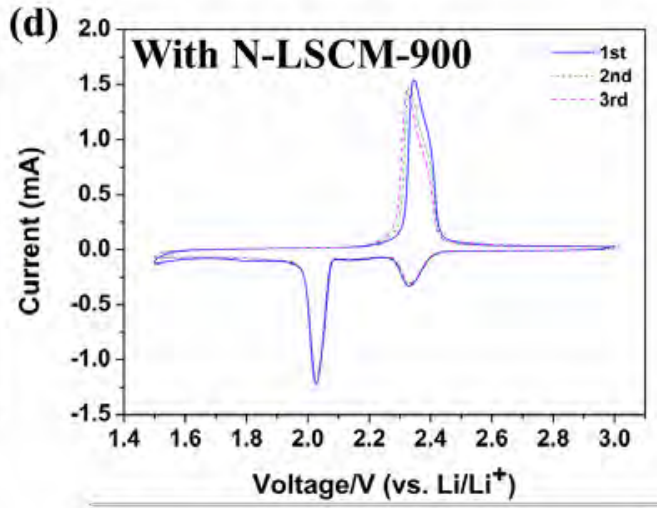

(e)

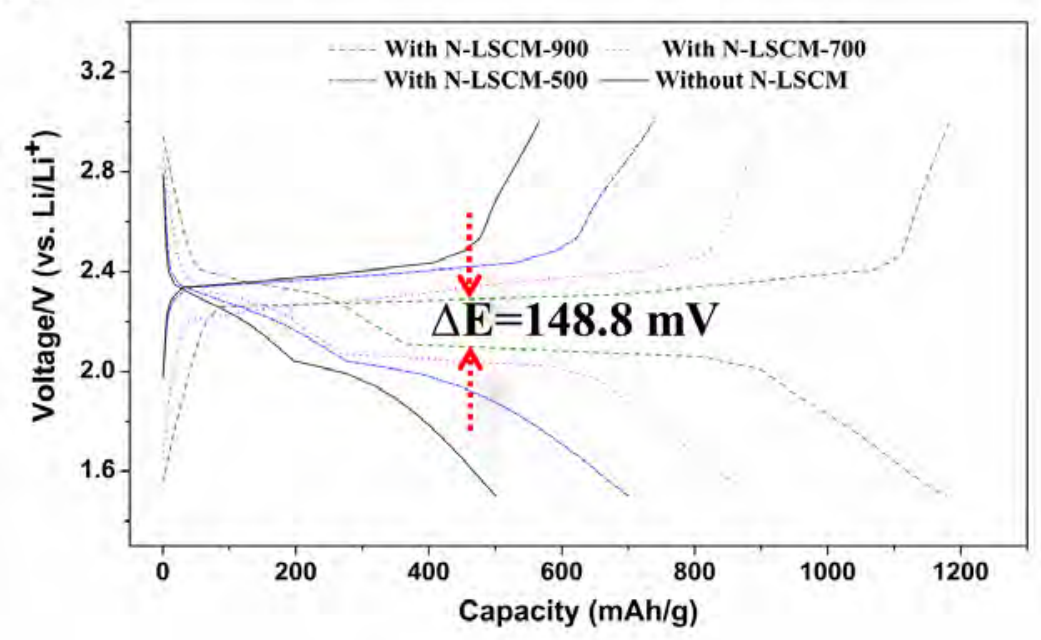

Figure $3 \mathrm{CV}$ curves of the cells without N-LSCM (a), with N-LSCM-500 (b), with N-LSCM-700 (c) and with N-LSCM-900 (d) and a scan rate of $0.1 \mathrm{mV} \mathrm{s}^{-1}$; and galvanostatic charge/discharge profiles of the Li-S batteries at the current of $1675 \mathrm{~mA} \mathrm{~g}^{-1}$ without N-LSCM, with N-LSCM-500, N-LSCM-700 and N-LSCM-900 (e), and cut-off voltage is between 1.5 to $3.0 \mathrm{~V}$.

The cycling performances of the sulfur electrodes with and without N-LSCM were tested at $1675 \mathrm{~mA} \mathrm{~g}^{-1}$, as shown in Figure 4a. The sulfur electrode with N-LSCM-900 delivers an initial discharge capacity as high as $1179.3 \mathrm{~mA} \mathrm{~h} \mathrm{~g}^{-1}$ (normalized to $\mathrm{S}$, the same hereafter), 
which is much higher than the electrode without the N-LSCM (only $508.7 \mathrm{~mA} \mathrm{~h} \mathrm{~g}^{-1}$ ). After several cycles for activation, a maximum capacity of $1212 \mathrm{~mA} \mathrm{~h} \mathrm{~g}^{-1}$ reaches for the sulfur electrode with N-LSCM-900. After 500 deep charge/discharge cycles, a reversible discharge capacity of $623.6 \mathrm{~mA} \mathrm{~h} \mathrm{~g}^{-1}$ is obtained with a fading rate of $0.09 \%$ cycle $^{-1}$; following the sulfur electrode with N-LSCM-700 maintains a reversible capacity of $466.5 \mathrm{~mA} \mathrm{~h} \mathrm{~g}^{-1}$. While capacities of the sulfur electrodes with N-LSCM-500 and the pure sulfur electrodes decrease quickly to $\approx 263.5$ and $89.4 \mathrm{~mA} \mathrm{~h} \mathrm{~g}^{-1}$, respectively. What's more, the coulombic efficiencies of the cells with the N-LSCM-900 interlayers increase to nearly $100 \%$ from $89 \%$ of the cells without the N-LSCM interlayer, which means the shuttle phenomenon has been effectively prohibited $^{4,53}$. Compared the cycling performances of Li-S batteries with different biochar blocking layers in Table S4, the Li-S batteries with N-LSCM-900 interlayer shows the best long cycling performances at high discharge rate. Moreover, the comparison between the asprepared carbon-sulfur composite and other $\mathrm{N}$-doped porous carbon-sulfur composites in the Table S5 shows that the performance of our product in terms of initial capacity, reversible capacity and rate capability is significantly superior to others even when the sulfur content reach as high as $70 \%$.

Aiming to examine the N-LSCM insertion could enhance the tolerance to high current rates, the batteries with and without the N-LSCM were cycled at stepwise current rates as shown in Figure $4 \mathrm{~b}$. It is undoubted that the cells with the N-LSCM reveal better rate capabilities compared to that without the N-LSCM. And with the annealed temperature increasing of the LS, the batteries with relative N-LSCM shows increasing rate capabilities. Thus the batteries with N-LSCM-900 demonstrates the best performances, i.e., after activated at the low currents (335 $\mathrm{mA} \mathrm{g}^{-1}$ ) for five cycles, a highly reversible capacity of $659.4 \mathrm{~mA} \mathrm{~h} \mathrm{~g}$ is obtained at $3350 \mathrm{~mA} \mathrm{~g}^{-1}$, while $594.5 \mathrm{~mA} \mathrm{~h} \mathrm{~g}^{-1}$ is also achieved when the current rate is set at as high as $6700 \mathrm{~mA} \mathrm{~g}^{-1}$. The capacity recovers to $783.8 \mathrm{~mA} \mathrm{~h} \mathrm{~g}^{-1}$ when switching the current rate back 
to $837.5 \mathrm{~mA} \mathrm{~g}^{-1}$, indicating excellent electrode integrity and fast reaction kinetics in the batteries with N-LSCM- $900^{54}$.
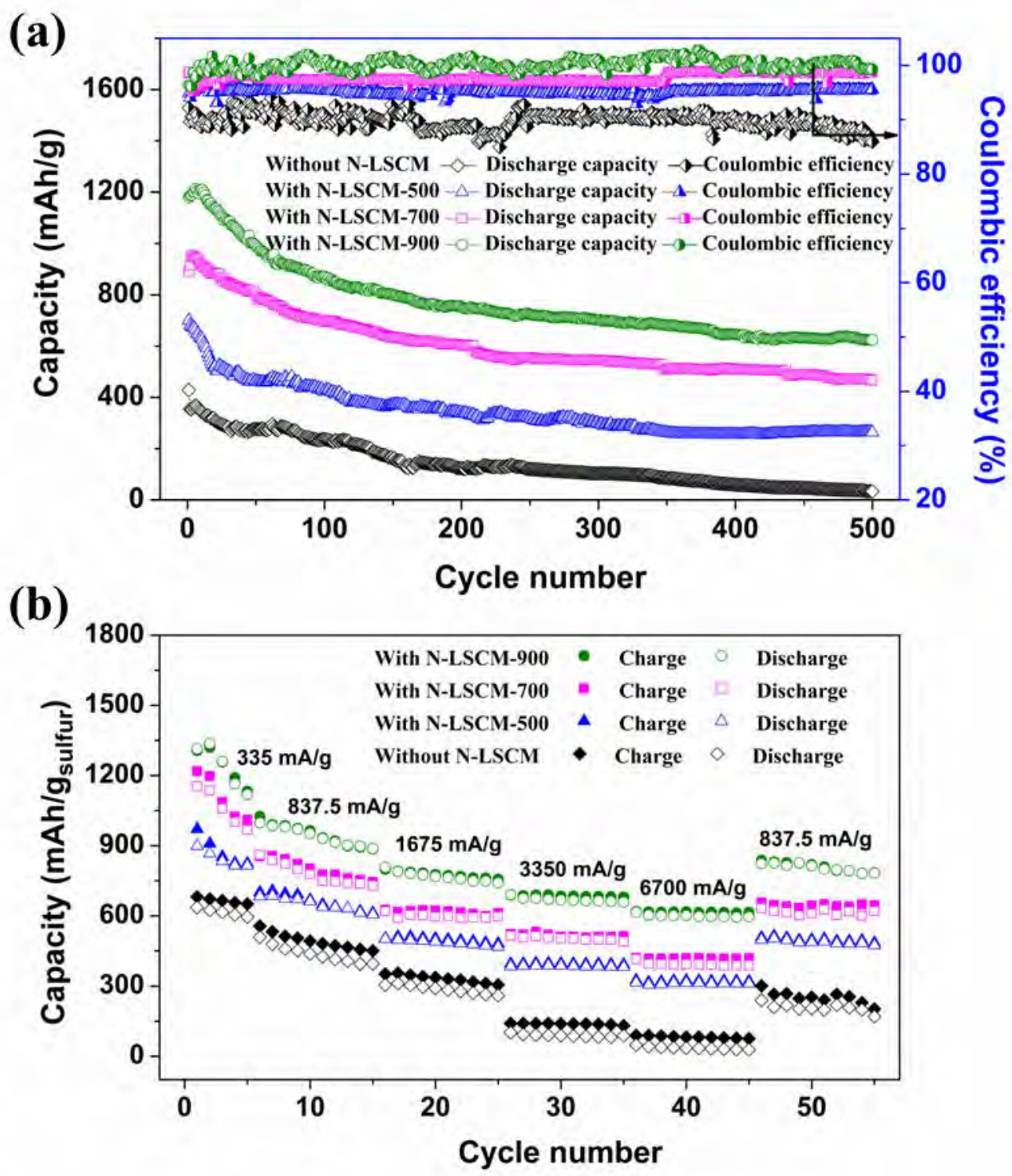

Figure 4 (a) Cycle life of the Li-S cells with and without N-LSCM interlayers at $1675 \mathrm{~mA} \mathrm{~g}^{-1}$ and (b) Rate capability of the Li-S cells with and without N-LSCM interlayers.

To further explore the mechanisms of the enhanced electrochemical performances of the sulfur electrode with N-LSCM interlayer, electrochemical impedance spectroscopy (EIS) measurement is performed before discharge and after 100 deep discharges. The Nyquist plots of the cells before and after cycling have been demonstrated in Figure S4 as well as the corresponding equivalent circuit. Before cycling, only a single depressed semicircle in the 
high-to-medium frequency region and an inclined line in the low frequency region can be observed in Figure 4a, in line with the charge-transfer resistance $\left(R_{\mathrm{ct}}\right)$ and the Warburg impedance $\left(W_{0}\right)^{4,25,49-50}$, respectively. And the ohmic resistance $\left(R_{\mathrm{e}}\right)$ of the cell, including the electrolyte and electrode resistances, corresponds to the high-frequency intercept on the real axis $^{4,}{ }^{49-50}$. While after cycling 100 cycles, two semicircles (Figure S4b) appears in the Nyquist plot. The one in the high-frequency region reflects the passivation film resistance $\left(R_{\mathrm{s}}\right)$, which is contributed by the $\mathrm{Li}_{2} \mathrm{~S}$ (or $\mathrm{Li}_{2} \mathrm{~S}_{2}$ ) layers on the surface of the electrode ${ }^{4,25,55}$, while the other one in the medium-frequency range also reflects the $R_{\mathrm{ct}}{ }^{4,25,55}$. The $R_{\mathrm{e}}, R_{\mathrm{s}}, R_{\mathrm{ct}}$ values of the cells with different interlayers acquired from the equivalent circuits are shown in Table S6. As expectedly, the $R_{c t}$ of the cell without the N-LSCM interlayer is obviously higher than that of the cells with the N-LSCM interlayer before discharge, which is due to the enhanced conductivity after the conductive N-LSCM interlayer is inserted in the cells. And the $R_{c t}$ of the cells with the N-LSCM-900 interlayers is even smaller than the batteries with N-LSCM700 and N-LSCM-500 interlayers, consistent with the results from the four-point probe conductivity measurement. This refers to the aforementioned reasons, i.e., the higher specific surface area facilities more activation reaction sites thus boost the active materials utilization and more pores as well as the higher conductivity is beneficial to Li ions mass transport and electrons transfer ${ }^{22}$. After 100 deep discharges, due to the infiltration of the electrolyte, chemical activation and active materials dissolution and redistribution ${ }^{4,22,56}$, all the $R_{c t}$ values decrease significantly, which also indicates that the formation of the SEI film on the surface of the electrode facilitates the Li ions transportation easier ${ }^{22,50,57}$. Regarding to the $R_{S}$, the cells with the N-LSCM interlayer are far smaller than those of the cells without the N-LSCM interlayer, further demonstrating that the N-LSCM interlayer could efficiently restrain the polysulfides from shuttling to form the $\mathrm{Li}_{2} \mathrm{~S}_{2} / \mathrm{Li}_{2} \mathrm{~S}$ film ${ }^{4,22}$. Especially, the cell with the NLSCM-900 interlayer has smallest $R_{c t}$ and $R_{S}$ values, which may be due to the highest conductivity, specific surface area and pore volume of the LSC-900. 
Following we investigated the electrochemical performances of the Li-Se batteries with the N-LSCMs as the blocking layers (Figure 5). Figure 5a shows the CV curves of the Li-Se batteries without and with N-LSCM-900. For the batteries with N-LSCM-900 blocking layer, in the first cathodic scan, two sharp reduction peaks at $2.1 \mathrm{~V}$ and $1.95 \mathrm{~V}$ are observed, corresponding to the stepwise electrochemical reduction of selenium to polyselenides and finally to $\mathrm{Li}_{2} \mathrm{Se}^{8}$. And one strong and sharp oxidation peak at $2.25 \mathrm{~V}$ appears in the subsequent anodic scan, which is in accordance with reversible conversion of $\mathrm{Li}_{2} \mathrm{Se}$ to high order polyselenides or even elemental selenium ${ }^{8}$. Meanwhile it is observed that the cathodic and anodic peaks are nearly overlapped after the first cycle, demonstrating the good reversibility and stability of the cell ${ }^{2}$. And the two distinguished cathodic peaks are line with the discharge plateaus in Figure 5b. For comparison, the CV curves of the cell without NLSCM (Figure S5a) were also conducted. The wider peaks and lower current with larger polarization indicated a slow kinetic process during cycling ${ }^{2}$. Moreover, the redox peak profile of Se electrode with N-LSCM-900 was more symmetric and sharper than that of Se electrode without N-LSCM-900, demonstrating that the redox kinetics were enhanced due to the reduced diffusion length of lithium ${ }^{3}$. The galvanostatic charge/discharge voltage profiles of the Se cathode with N-LSCM-900 in the different cycles are shown in Figure 5b. Two voltage plateaus at $2.15 \mathrm{~V}$ and $1.97 \mathrm{~V}$ in the discharge process correspond to the reduction of selenium to different chain length polyselenides ${ }^{8}$. One very long plateaus located at $2.10 \mathrm{~V}$ to 2.20 $\mathrm{V}$ are observed in the charge process, corresponding to the reverse reactions from $\mathrm{Li}_{2} \mathrm{Se}$ to elemental selenium ${ }^{8}$. Such voltage plateaus are consistent well with the CV measurements. Moreover, the voltage gap between charge and discharge plateaus at the initial cycle of Se electrode with N-LSCM-900 $(158.3 \mathrm{mV})$ is much smaller than that of Se electrode without NLSCM (336.0 mV, Figure S5b). Particularly even after 200 cycles, the Se electrode with NLSCM-900 still illustrates obvious two voltage plateaus and changed thinly at such high charge/discharge currents; while it is difficult to observe the voltage plateaus of Se electrode 
without N-LSCM, which means the low polarization of the Se electrodes with N-LSCM and further implies the high electrochemical stability and reversibility of the Se electrode with NLSCM- $900^{8}$.

Figure 5c shows the long cycle performance of Se electrodes with and without N-LSCM at a high current density of $1356 \mathrm{~mA} \mathrm{~g}^{-1}$. Undoubtedly, the Li-Se batteries with the N-LSCM demonstrate better cycling performances compared to that without the N-LSCM. Similar as the results of $\mathrm{Li}-\mathrm{S}$ batteries, the $\mathrm{Li}-\mathrm{Se}$ batteries with $\mathrm{N}-\mathrm{LSCM}-900$ gives the best performances compared to that with N-LSCM-700 and N-LSCM-500, which the Se electrode with N-LSCM-900 could deliver an exceptional high capacity of approximately $350 \mathrm{~mA} \mathrm{~h} \mathrm{~g}{ }^{-1}$ (normalized to Se, the same hereafter), with nearly $100 \%$ coulombic efficiency throughout the 1000 cycles cycling. At the same time, the Se electrode with N-LSCM-900 also demonstrates excellent high-rate capability, as shown in Figure 5d. When the current rate increases from 135.6 to $339,678,1356$, and $3390 \mathrm{~mA} \mathrm{~g}^{-1}$, the electrode displays good capacity retention varying from 655.4 to $613.4,572.8,504.5$, and $358.4 \mathrm{~mA} \mathrm{~h} \mathrm{~g}^{-1}$, respectively. When the current rate returns to $339 \mathrm{~mA} \mathrm{~g}^{-1}$, the capacity can recover to $599.7 \mathrm{~mA} \mathrm{~h} \mathrm{~g}^{-1}$, indicating the excellent electrochemical reversibility of the Se electrode with N-LSCM-900 interlayer ${ }^{8}$. Compared to the Se electrode with N-LSCM-900, the Se electrodes with N-LSCM-700 and N-LSCM-500 exhibit poorer rate performances. And similar as the Li-S batteries, the pure Se electrodes without the N-LSCM shows the worst rate performances too, which suggests that N-LSCM interlayer could remarkably prohibit the polyselenides shuttling, improve active materials utilization and accelerate the kinetics. 
(a)

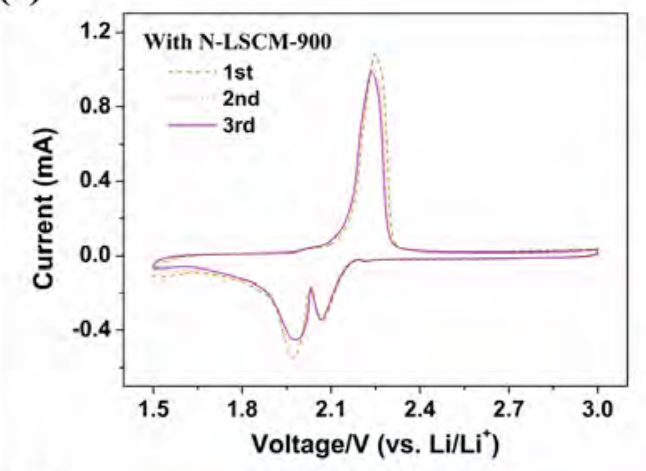

(b)

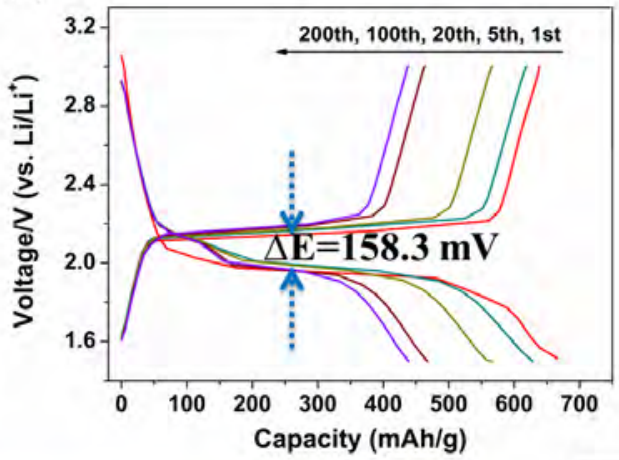

(c)
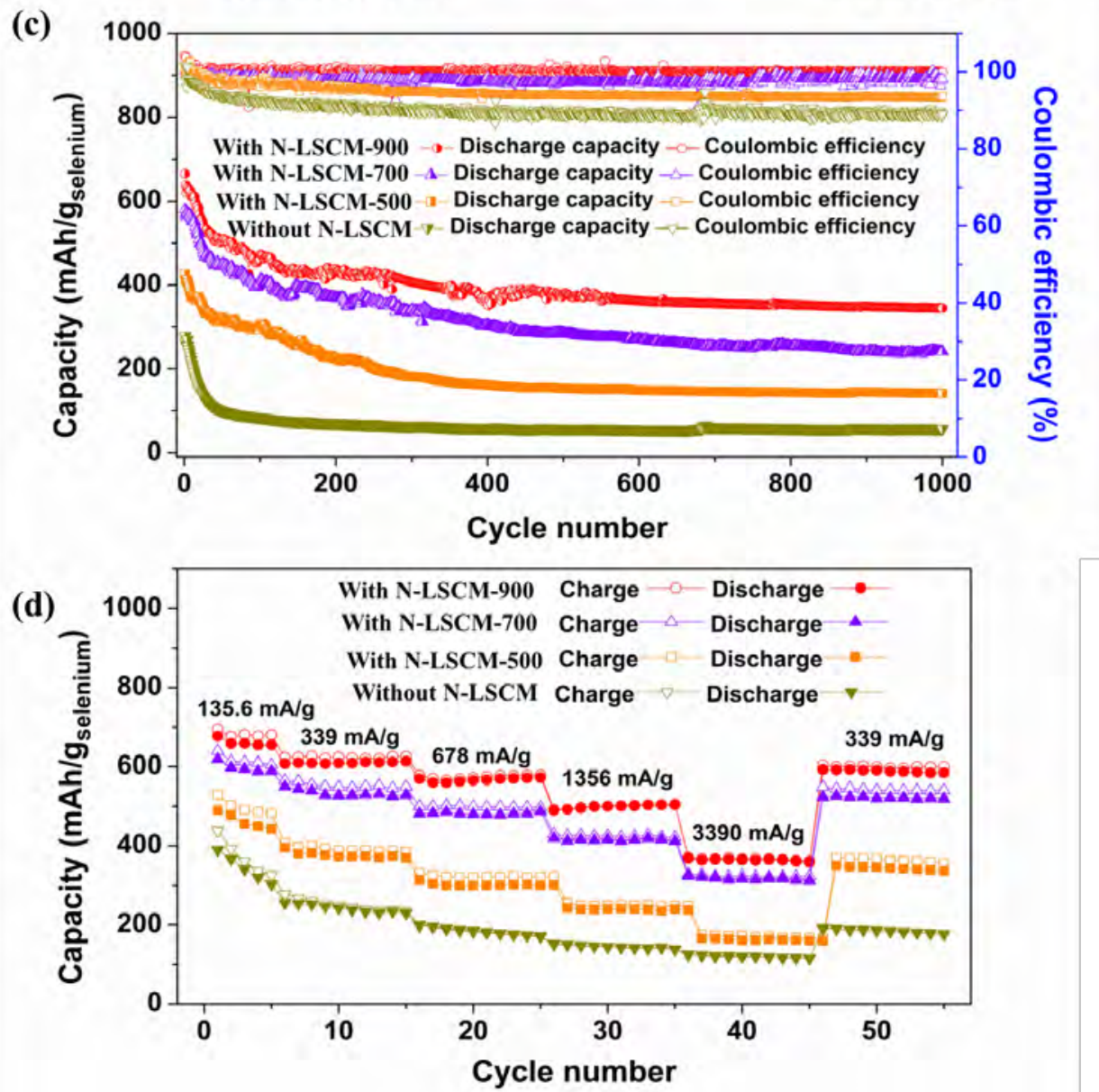

Figure 5 (a) CV curves of the Li-Se cells with N-LSCM-900, scan rate is $0.1 \mathrm{mV} \mathrm{s}^{-1}$; (b) Galvanostatic charge/discharge profiles of the Li-Se batteries at the current of $1356 \mathrm{~mA} \mathrm{~g}^{-1}$ with N-LSCM-900, cut-off voltage is between 1.5 to $3.0 \mathrm{~V}$; (c) Cycle life of the Li-Se cells with and without N-LSCM interlayer at the current of $1356 \mathrm{~mA} \mathrm{~g}^{-1}$ and (d) Rate capability of the Li-Se cells with and without N-LSCM interlayers.

The electrochemical impedance spectroscopy (EIS) measurements before discharge and after 100 deep discharges for the Li-Se batteries have also been conducted. Similar to the previous literatures ${ }^{28,58}$, only a single depressed semicircle in the high-to-medium frequency 
region and an inclined line in the low frequency region can be observed in the Nyquist plots of these Se electrodes no matter before discharge or after 100 cycles (Figure S6). The semicircle corresponds to the internal resistance of the cathode including bulk impedance and interfacial impedance between the electrode and the electrolyte ${ }^{58}$. The inclined line at low frequency reflects the $\mathrm{Li}$ ion diffusion into the active mass ${ }^{28,58}$. It can be seen in Figure S6 and Table S7, the charge transfer resistance of the cells with the N-LSCM decreases significantly by the insertion of the N-LSCM interlayer. This is because that the porous NLSCM can provide a conductive network between elemental Se, and thus decreases the inner resistance of battery ${ }^{58}$. Herein, by serving efficient electron migration pathways to and from the active material, the N-LSCM interlayer for the Se cathode can improve the active material utilization and thereby raising the specific capacity of the cell ${ }^{28}$.

What's more, the N-LSCM was employed as the lithium polyiodides adsorbent for $\mathrm{Li}-\mathrm{I}_{2}$ batteries. Interestingly, different from the previous carbon-iodine cathodes that display typical two pairs of oxidation-reduction peaks ${ }^{12-13}$, no cathodic peaks can be observed in the $\mathrm{Li}-\mathrm{I}_{2}$ batteries with the N-LSCM interlayer as shown in Figure 6a, which suggests the pseudocapacitive charge/discharge behavior of the $\mathrm{I}_{2}$ cathode matching with the N-LSCM interlayer ${ }^{59-60}$. Moreover, it can't be observed the discharge plateaus from the charge/discharge profiles of the $\mathrm{Li}-\mathrm{I}_{2}$ batteries with the N-LSCM-900 interlayer in Figure $6 \mathrm{~b}$, also indicating the typical capacitive charge/discharge behavior ${ }^{60}$. The lithium storage performances of the $\mathrm{Li}-\mathrm{I}_{2}$ batteries with the N-LSCM interlayer at $2110 \mathrm{~mA} \mathrm{~g}^{-1}$ (2.0 to $3.8 \mathrm{~V}$ ) are shown in Figure 6c. In comparison with the cycling performances of the pure $\mathrm{I}_{2}$ electrode and the N-LSCM-900 cathode in Figure S7, the N-LSCM interlayer remarkably improves the charge/discharge capacity and the coulombic efficiency of the $\mathrm{Li}-\mathrm{I}_{2}$ batteries. In particular, the $\mathrm{I}_{2}$ electrode with N-LSCM-900 interlayer delivers a reversible capacity of $207.7 \mathrm{~mA} \mathrm{~h} \mathrm{~g}^{-1}$ that approaches the theory specific capacity $\left(211 \mathrm{~mA} \mathrm{~h} \mathrm{~g}^{-1}\right)$. Further when the rate increases to as high as $10550 \mathrm{~mA} \mathrm{~g}^{-1}$, it can still remain a fantastic reversible capacity of around 150 
$\mathrm{mA} \mathrm{h} \mathrm{g}^{-1}$ after ultra-long 5000 cycles. Such amazing performances could be attributed to the porous structure and high surface area of N-LSCM that can not only facilitate the fast transport of ions throughout the entire materials and significantly shorten transportation pathways $^{60}$, but also can physically adsorb the iodides to reduce the shuttle phenomenon. In addition, the nitrogen heteroatoms may contribute to adsorbing the iodides.
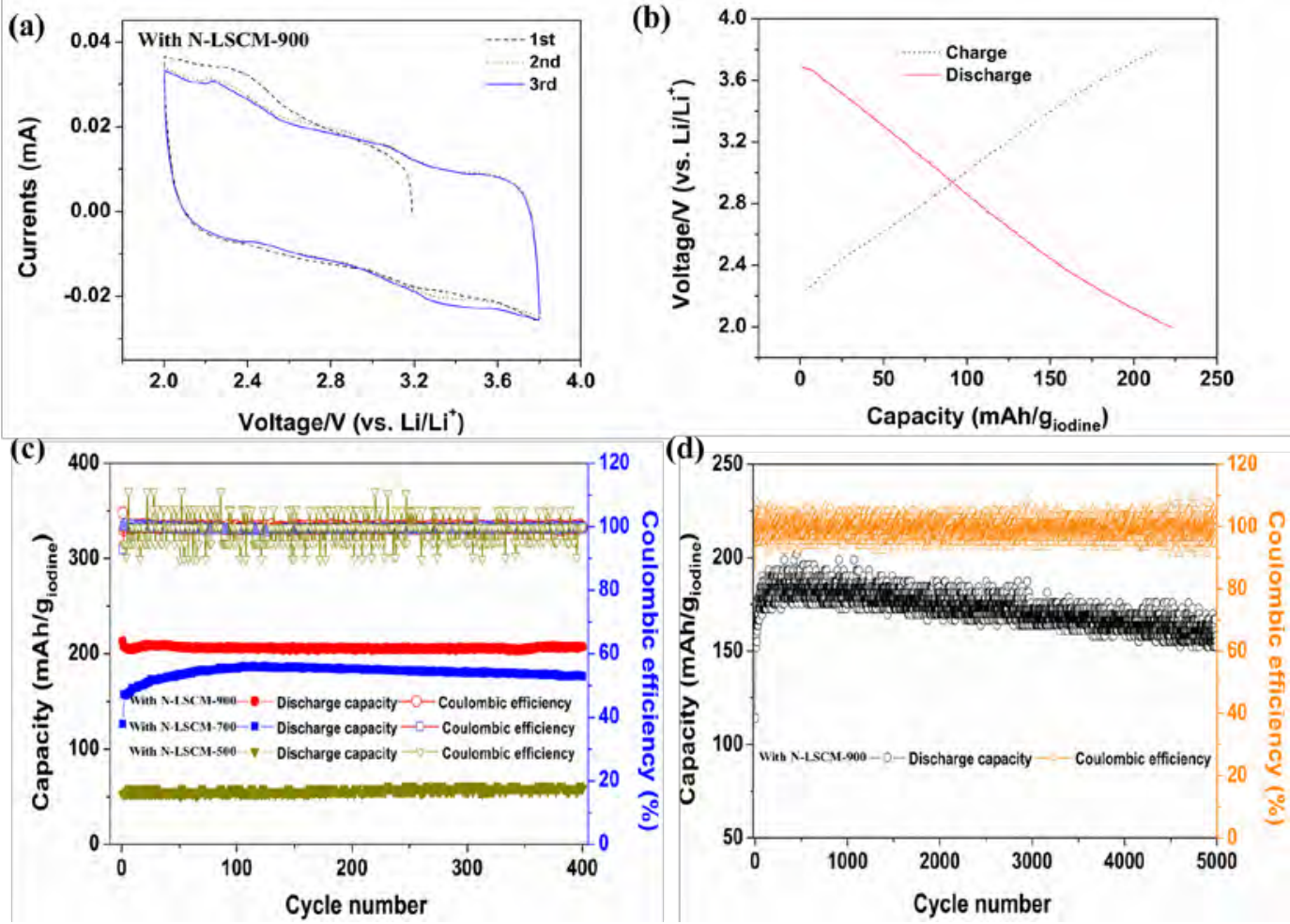

Figure 6 (a) CV curves of the $\mathrm{Li}-\mathrm{I}_{2}$ cells with N-LSCM-900, scan rate is $0.1 \mathrm{mV} \mathrm{s}{ }^{-1}$; (b) Galvanostatic charge/discharge profiles of the $\mathrm{Li}-\mathrm{I}_{2}$ cells at the current of $2110 \mathrm{~mA} \mathrm{~g}^{-1}$ with N-LSCM-900, cut-off voltage is between 2.0 to $3.8 \mathrm{~V}$; (c) Cycle life of the $\mathrm{Li}-\mathrm{I}_{2}$ cells with $\mathrm{N}-\mathrm{LSCM}$ interlayer at the current of $2110 \mathrm{~mA} \mathrm{~g}^{-1}$, and (d) the ultra-long cycle life of the $\mathrm{Li}-\mathrm{I}_{2}$ cells with N-LSCM-900 interlayer at the high current of $10550 \mathrm{~mA} \mathrm{~g}^{-1}$.

The aforementioned materials and electrochemical characterization have experimentally demonstrated the N-LSCM can be an effective universal blocking layer for the batteries. What's more, the adsorption experiments as shown in Figure S8 obviously demonstrate that the N-LSCM could effectively adsorb $\mathrm{Li}_{2} \mathrm{~S}_{8}, \mathrm{Li}_{2} \mathrm{Se}_{8}$ and $\mathrm{LiI}_{3}$ individually in the solution. In order to verify the polysulfides, polyselenides and polyiodides have been intercepted by the blocking layer N-LSCM, the SEM and element mapping for the N-LSCM-900 after washing with acetone has been conducted. As shown in the Figure S9, the active materials have filled 
in the pores and distributed on the surface of the N-LSCM-900 after charge/discharge for 100 cycles, while the element mapping proved the elements S, Se and I are distributed evenly in the N-LSCM-900. This confirms the N-LSCM interlayer can effectively retain the polysulfides, polyselenides and polyiodides. More importantly, due to the as-prepared interlayer has excellent conductivity, as well as large geometric and surface area that could not only effectively prohibit the polysulfides from shuttling to the lithium anode, but also bestow uniform current distribution in the lithium anode. This contributes to cyclability improvement of the $\mathrm{Li}$ anode and dendrite prevention.

In order to understand the role of nitrogen in N-LSC, the first-principle calculations were carried out on interaction between the Li clusters and the N-LSC. All the possible lithium based anions on the nitrogen doped LSC have been considered in the determination of the most stable configuration. The XPS analysis suggests that the N-LSC contains three types of nitrogen, which are the same as the nitrogen doped graphene (pyrrolic N, pyridinic N, graphitic N). Therefore, In the following, these three different types of $\mathrm{N}$ are considered to distinguish the their role as the blocking layer ${ }^{4}$. In order to evaluate the relative stability between the different configuration and compositions, the adsorption energy $\left(E_{a}\right)$ of Li cluster on the carbon framework with three different kinds of N-doping are calculated by firstprinciple calculations. $E_{a}$ is calculated using the following equation:

$$
E_{a}=E_{\text {total }}-E_{C}-E_{y}\left(y=\mathrm{Li}_{2} \mathrm{~S}_{8}, \mathrm{Li}_{2} \mathrm{Se}_{8}, \mathrm{LiI}_{3}\right)
$$

Here $E_{\text {total }}, E_{C}$, and $E_{y}$ are the energies of the whole system, carbon substrate, and polymer molecules, respectively. Table 1 illustrates the calculated $E_{a}$ on different active sites of the nitrogen doped LSC and Figure S10 shows the optimized adsorption structures. It is known that the more negative value of $E_{a}$ indicates the stronger adsorption capability. As shown in Table 1, among the three types of $\mathrm{N}$, the pyrrolic $\mathrm{N}$ shows the strongest ability to adsorb the typical clusters, such as $\mathrm{Li}_{2} \mathrm{~S}_{8}, \mathrm{Li}_{2} \mathrm{Se}_{8}$ and $\mathrm{LiI}_{3}$. Particularly, the adsorption energies of $\mathrm{LiI}_{3}$ $(1.75 \mathrm{eV})$ and $\mathrm{Li}_{2} \mathrm{Se}_{8}(1.60 \mathrm{eV})$ on the pyrrolic $\mathrm{N}$ is obviously larger than that of $\mathrm{Li}_{2} \mathrm{~S}_{8}(1.14$ 
eV). Such results clearly indicated that the N-LSC have the quite large adsorption ability for polysulfides, polyselenides and iodides, which is responsible for the confinement of the active materials within the cathodic chamber. More interestingly, the relative larger adsorption energies for iodides and polyselenides than polysulfidesis coincide with the higher and more stable cycling performances of $\mathrm{Li}-\mathrm{I}_{2}$ and $\mathrm{Li}-\mathrm{Se}$ batteries than the $\mathrm{Li}-\mathrm{S}$ batteries.

Table 1 The calculated adsorption energies $\left(E_{a}\right)$ for various lithium cluster based anions on different active sites of the N-LSC.

\begin{tabular}{cccc}
\hline \multirow{2}{*}{ Target } & \multicolumn{3}{c}{ Adsorption Energy $E_{a}(\mathrm{eV})$} \\
\cline { 2 - 4 } & Graphitic N & Pyridinic N & Pyrrolic N \\
\hline $\mathrm{Li}_{2} \mathrm{~S}_{8}$ & -0.57 & -0.84 & -1.14 \\
$\mathrm{Li}_{2} \mathrm{Se}_{8}$ & -0.93 & -0.99 & -1.60 \\
$\mathrm{LiI}_{3}$ & -0.87 & -1.05 & -1.75
\end{tabular}

According to the above experimental and calculation results, the significantly improved active materials utilization, cyclic stability, and high rate performance could be attributed to the unique hierarchical porous nanostructure, physical and chemical properties, in which (i) the excellent conductivity of N-LSC can facilitate electron transfer; (ii) the N-LSC can host large amounts of active materials and accommodate the electrode volume expansion; (iii) the abundant pores of N-LSC can effectively entrap active materials by physical adsorption and improves the energy density of the whole cathode; (iv) the hierarchical pores can provide short lithium-ion diffusion distance, enabling rapid ion transport and electrolyte diffusion; and (v) the hetero-doped nitrogen atoms show better affinity to polysulfides, polyselenides and iodides.

\section{CONCLUSIONS}

In summary, we have successfully synthesized the N-LSC with ultra-high conductivity, specific surface area and pore volume. Then the N-LSC is fabricated to N-LSC membrane and 
employed as a universal blocking layer for the cathode of $\mathrm{Li}-\mathrm{S}, \mathrm{Li}-\mathrm{Se}$ and $\mathrm{Li}-\mathrm{I}_{2}$ batteries. The resultant batteries deliver outstanding cycling performances and rate capabilities. In particular, with the N-LSCM-900 interlayer, the pure sulfur cathode delivers excellent cycling performance up to 500 cycles with a low decay rate of $0.094 \%$ and superior rate performance up to $6700 \mathrm{~mA} \mathrm{~g}^{-1}$; and the pure Se cathode with N-LSCM-900 displays outstanding cycling performance up to 1000 cycles with a very low decay rate of $0.048 \%$ per cycle and superior rate performance up to $3390 \mathrm{~mA} \mathrm{~g}^{-1}$; as well as the pure $\mathrm{I}_{2}$ cathode with N-LSCM-900 interlayer demonstrates an amazing cycling performance of $150 \mathrm{~mA} \mathrm{~h} \mathrm{~g}^{-1}$ at $10550 \mathrm{~mA} \mathrm{~g}^{-1}$ after 5000 cycles. The outstanding performance is attributed to the multi-functions offered by the N-LSCM. The N-LSCM facilitates the electron transport, accommodates the volume change of the electrodes, and prevents polysulfides, polyselenides, and iodides from shuttling to the anode region. The first-principle calculations further confirm the $\mathrm{N}$ atoms offer adsorption capability to critical active materials and intermediates such as $\mathrm{Li}_{2} \mathrm{~S}_{8} \mathrm{Li}_{2} \mathrm{Se}_{8}$ and $\mathrm{LiI}_{3}$. Overall, the as-prepared N-LSCM is a promising multifunctional blocking layer for the cathode of rechargeable lithium batteries in practical applications. Furthermore, the concept of such hierarchical pore architecture, conductive, cost-effective blocking layers would be easily expanded to other advanced energy storage systems, such as sulfur for $\mathrm{Na}-\mathrm{S}$ batteries, selenium for $\mathrm{Na}-\mathrm{Se}$ batteries and so on.

\section{ASSOCIATED CONTENT}

The Supporting Information is available free of charge on the ACS Publications website.

\section{AUTHOR INFORMATION}

\section{Corresponding Authors}

s.zhang@griffith.edu.au; $\underline{\text { hou@,pku.edu.cn; }}$ limin.liu@,csrc.ac.cn

\section{Notes}


The authors declare no competing financial interest.

\section{ACKNOWLEDGMENTS}

The authors acknowledge the financial support of the ARC Discovery Grants from the Australian Research Council, NSFC-RGC Joint Research Scheme (51361165201), NSFC (51125001, 51172005), Doctoral Program of the Ministry of Education of China (20120001110078), Opeing Funds of National Laboratory of Molecular Science and Key Laboratory of Functional Inorganic Material Chemistry (Heilongjiang University), Ministry of Education of China. And the authors also want to thank Limin Weng and Yang Chen for their help on drawing the scheme.

\section{REFERENCES}

1. Xu, Y.; Wen, Y.; Zhu, Y.; Gaskell, K.; Cychosz, K. A.; Eichhorn, B.; Xu, K.; Wang, C., Confined Sulfur in Microporous Carbon Renders Superior Cycling Stability in Li/S Batteries. Adv. Funct.Mater. 2015, 25, 4312-4320.

2. Fang, R.; Zhou, G.; Pei, S.; Li, F.; Cheng, H.-M., Localized polyselenides in a graphene-coated polymer separator for high rate and ultralong life lithium-selenium batteries. Chem. Commun. 2015, 51, 3667-3670.

3. Zeng, L. C.; Zeng, W. C.; Jiang, Y.; Wei, X.; Li, W. H.; Yang, C. L.; Zhu, Y. W.; Yu, Y., A Flexible Porous Carbon Nanofibers-Selenium Cathode with Superior Electrochemical Performance for Both Li-Se and Na-Se Batteries. Adv. Energy Mater. 2015, 5, $1401377-$ 1401387.

4. Gu, X.; Tong, C.-J.; Lai, C.; Qiu, J.; Huang, X.; Yang, W.; Wen, B.; Liu, L.-M.; Hou, Y.; Zhang, S., Porous Nitrogen and Phosphorous Dual Doped Graphene Blocking Layer for High Performance Li-S Batteries. J. Mater. Chem. A 2015, 3, 16670-16678.

5. Gu, X.; Wang, Y.; Lai, C.; Qiu, J.; Li, S.; Hou, Y.; Martens, W.; Mahmood, N.; Zhang, S., Microporous bamboo biochar for lithium-sulfur batteries. Nano Res. 2015, 8, 129-139.

6. Yin, Y. X.; Xin, S.; Guo, Y. G.; Wan, L. J., Lithium-Sulfur Batteries: Electrochemistry, Materials, and Prospects. Angew. Chem. Int. Ed. Engl. 2013, 52, 13186-200.

7. Xin, S.; Gu, L.; Zhao, N. H.; Yin, Y. X.; Zhou, L. J.; Guo, Y. G.; Wan, L. J., Smaller Sulfur Molecules Promise Better Lithium-Sulfur Batteries. J. Am. Chem. Soc. 2012, 134, 18510-18513.

8. Han, K.; Liu, Z.; Shen, J.; Lin, Y.; Dai, F.; Ye, H., A Free-Standing and Ultralong-Life Lithium-Selenium Battery Cathode Enabled by 3D Mesoporous Carbon/Graphene Hierarchical Architecture. Adv. Funct.Mater. 2015, 25, 455-463.

9. Lee, J. T.; Kim, H.; Oschatz, M.; Lee, D.-C.; Wu, F.; Lin, H.-T.; Zdyrko, B.; Cho, W. I.; Kaskel, S.; Yushin, G., Micro- and Mesoporous Carbide-Derived Carbon-Selenium Cathodes for High-Performance Lithium Selenium Batteries. Adv. Energy Mater. 2015, 5, 14009811400987. 
10. Yang, C. P.; Yin, Y. X.; Guo, Y. G., Elemental Selenium for Electrochemical Energy Storage. J. Phys. Chem. Lett. 2015, 6, 256-66.

11. Zhao, Y.; Hong, M.; Bonnet Mercier, N.; Yu, G.; Choi, H. C.; Byon, H. R., A 3.5 V Lithium-Iodine Hybrid Redox Battery With Vertically Aligned Carbon Nanotube Current Collector. Nano Lett. 2014, 14, 1085-1092.

12. Zhao, Q.; Lu, Y.; Zhu, Z.; Tao, Z.; Chen, J., Rechargeable Lithium-Iodine Batteries with Iodine/Nanoporous Carbon Cathode. Nano Lett. 2015, 15, 5982-5987.

13. Wang, Y. L.; Sun, Q. L.; Zhao, Q. Q.; Cao, J. S.; Ye, S. H., Rechargeable Lithium/Iodine Battery with Superior High-Rate Capability by Using Iodine-Carbon Composite as Cathode. Energy Environ. Sci. 2011, 4, 3947-3950.

14. Peng, X.; Wang, L.; Zhang, X.; Gao, B.; Fu, J.; Xiao, S.; Huo, K.; Chu, P. K., Reduced Graphene Oxide Encapsulated Selenium Nanoparticles for High-power Lithium-Selenium Battery Cathode. J. Power Sources 2015, 288, 214-220.

15. Gu, X. X.; Zhang, S. Q.; Hou, Y. L., Graphene-Based Sulfur Composites for Energy Storage and Conversion in Li-S Batteries. Chin. J. Chem. 2016, 34, 11-31.

16. Gao, X.; Li, J.; Guan, D.; Yuan, C., A Scalable Graphene Sulfur Composite Synthesis for Rechargeable Lithium Batteries with Good Capacity and Excellent Columbic Efficiency. ACS Appl. Mater. Interf. 2014, 6, 4154-4159.

17. Xu, T.; Song, J.; Gordin, M. L.; Sohn, H.; Yu, Z.; Chen, S.; Wang, D., Mesoporous carbon-carbon nanotube-sulfur composite microspheres for high-areal-capacity lithium-sulfur battery cathodes. ACS Appl Mater Interfaces 2013, 5 (21), 11355-62.

18. Li, G.-C.; Li, G.-R.; Ye, S.-H.; Gao, X.-P., A Polyaniline-Coated Sulfur/Carbon Composite with an Enhanced High-Rate Capability as a Cathode Material for Lithium/Sulfur Batteries. Adv. Energy Mater. 2012, 2 (10), 1238-1245.

19. Zhang, J.; Xu, Y.; Fan, L.; Zhu, Y.; Liang, J.; Qian, Y., Graphene-Encapsulated Selenium/Polyaniline Core-Shell Nanowires with Enhanced Electrochemical Performance for Li-Se Batteries. Nano Energy 2015, 13, 592-600.

20. Lai, C.; Wu, Z.; Gu, X.; Wang, C.; Xi, K.; Kumar, R. V.; Zhang, S., Reinforced Conductive Confinement of Sulfur for Robust and High-Performance Lithium-Sulfur Batteries. ACS Appl Mater Interfaces 2015, 7, 23885-23892.

21. Wang, H.; Zhang, C.; Chen, Z.; Liu, H. K.; Guo, Z., Large-Scale Synthesis of Ordered Mesoporous Carbon Fiber and Its Application as Cathode Material for Lithium-Sulfur Batteries. Carbon 2015, 81, 782-787.

22. Gu, X.; Lai, C.; Liu, F.; Yang, W.; Hou, Y.; Zhang, S., A Conductive Interwoven Bamboo Carbon Fiber Membrane for Li-S Batteries. J. Mater. Chem. A 2015, 3, 9502-9509.

23. Su, Y. S.; Manthiram, A., A New Approach to Improve Cycle Performance of Rechargeable Lithium-Sulfur Batteries by Inserting a Free-Standing MWCNT Interlayer. Chem. Commun. 2012, 48, 8817-8819.

24. Su, Y. S.; Manthiram, A., Lithium-Sulphur Batteries With a Microporous Carbon Paper as a Bifunctional Interlayer. Nat. Commun. 2012, 3, 1166-1171.

25. Zhou, G.; Pei, S.; Li, L.; Wang, D. W.; Wang, S.; Huang, K.; Yin, L. C.; Li, F.; Cheng, H. M., A Graphene-Pure-Sulfur Sandwich Structure for Ultrafast, Long-life Lithium-Sulfur Batteries. Adv. Mater. 2014, 26, 625-631.

26. Xiao, Z.; Yang, Z.; Wang, L.; Nie, H.; Zhong, M.; Lai, Q.; Xu, X.; Zhang, L.; Huang, S., A Lightweight $\mathrm{TiO}(2) /$ Graphene Interlayer, Applied as a Highly Effective Polysulfide Absorbent for Fast, Long-Life Lithium-Sulfur Batteries. Adv. Mater. 2015, 27, 2891-2898.

27. Zhang, K.; Li, Q.; Zhang, L.; Fang, J.; Li, J.; Qin, F.; Zhang, Z.; Lai, Y., From Filter Paper to Carbon Paper and Toward Li-S Battery Interlayer. Mater. Lett. 2014, 121, 198-201.

28. Zhang, Z.; Zhang, Z.; Zhang, K.; Yang, X.; Li, Q., Improvement of Electrochemical Performance of Rechargeable Lithium-Selenium Batteries by Inserting a Free-Standing Carbon Interlayer. RSC Adv. 2014, 4, 15489-15492. 
29. Huang, Y.; Zheng, M.; Lin, Z.; Zhao, B.; Zhang, S.; Yang, J.; Zhu, C.; Zhang, H.; Sun, D.; Shi, Y., Flexible Cathodes and Multifunctional Interlayers based on Carbonized Bacterial Cellulose for High-Performance Lithium-Sulfur Batteries. J. Mater. Chem. A 2015, 3, 1091010918.

30. Zhang, Y.; Zhao, Y.; Konarov, A.; Li, Z.; Chen, P., Effect of Mesoporous Carbon Microtube Prepared by Carbonizing the Poplar Catkin on Sulfur Cathode Performance in Li/S Batteries. J. Alloys Compd. 2015, 619, 298-302.

31. Chung, S. H.; Manthiram, A., A Natural Carbonized Leaf as Polysulfide Diffusion Inhibitor for High-Performance Lithium-Sulfur Battery Cells. ChemSusChem 2014, 7, 1655 1661.

32. Chung, S. H.; Manthiram, A., Carbonized Eggshell Membrane as a Natural Polysulfide Reservoir for Highly Reversible Li-S Batteries. Adv. Mater. 2014, 26, 1360-1365.

33. Qin, F. R.; Zhang, K.; Fang, J.; Lai, Y. Q.; Li, Q.; Zhang, Z. A.; Li, J., High Performance Lithium Sulfur Batteries with a Cassava-Derived Carbon Sheet as a Polysulfides Inhibitor. New J. Chem. 2014, 38, 4549-4554.

34. Yuan, Y.; Zhou, S.; Liu, Y.; Tang, J., Nanostructured Macroporous Bioanode Based on Polyaniline-Modified Natural Loofah Sponge for High-Performance Microbial Fuel Cells. Environ. Sci. Technol. 2013, 47, 14525-14532.

35. Luan, Y.; Wang, L.; Guo, S.; Jiang, B.; Zhao, D.; Yan, H.; Tian, C.; Fu, H., A Hierarchical Porous Carbon Material from a Loofah Sponge Network for High Performance Supercapacitors. RSC Adv. 2015, 5, 42430-42437.

36. Tanobe, V. O. A.; Sydenstricker, T. H. D.; Munaro, M.; Amico, S. C., A Comprehensive Characterization of Chemically Treated Brazilian Sponge-Gourds (Luffa Cylindrica). Polym. Test. 2005, 24, 474-482.

37. Kresse, G., Efficient Iterative Schemes for ab Initio Total-Energy Calculations Using a Plane-Wave Basis Set. Phys. Rev. B 1996, 54, 11169-11186.

38. Blochl, P. E., Projector Augmented-Wave Method. Phys. Rev. B 1994, 50, 1795317979.

39. Perdew, J. P.; Burke, K.; Ernzerhof, M., Generalized Gradient Approximation Made Simple. Phys. Rev. Lett. 1996, 77, 3865-3868.

40. Grimme, S.; Antony, J.; Ehrlich, S.; Krieg, H., A Consistent and Accurate ab Initio Parametrization of Density Functional Dispersion Correction (DFT-D) for the 94 Elements HPu. J. Chem. Phys. 2010, 132, 154104-154122.

41. Grimme, S.; Ehrlich, S.; Goerigk, L., Effect of the Damping Function in Dispersion Corrected Density Functional Theory. J. Comput. Chem. 2011, 32, 1456-1465.

42. Xiao, Y.; Chen, H.; Zheng, M.; Dong, H.; Lei, B.; Liu, Y., Porous Carbon with Ultrahigh Specific Surface Area Derived from Biomass Rice Hull. Mater. Lett. 2014, 116, 185-187.

43. Elmira Memarzadeh Lotfabad; Jia Ding; Kai Cui; Alireza Kohandehghan; Peter Kalisvaart; Michael Hazelton; Mitlin, D., High-Density Sodium and Lithium Ion Battery Anodes from Banana Peels. ACS NANO 2014, 8, 7115-7129.

44. Jiang, J.; Zhu, J. H.; Ai, W.; Fan, Z. X.; Shen, X. N.; Zou, C. J.; Liu, J. P.; Zhang, H.; Yu, T., Evolution of Disposable Bamboo Chopsticks into Uniform Carbon Fibers: a Smart Strategy to Fabricate Sustainable Anodes for Li-Ion Batteries. Energy Environ. Sci. 2014, 7, 2670-2679.

45. Song, J. X.; Gordin, M. L.; Xu, T.; Chen, S. R.; Yu, Z. X.; Sohn, H.; Lu, J.; Ren, Y.; Duan, Y. H.; Wang, D. H., Strong Lithium Polysulfide Chemisorption on Electroactive Sites of Nitrogen-Doped Carbon Composites For High-Performance Lithium-Sulfur Battery Cathodes. Angew. Chem. Int. Edit. 2015, 54, 4325-4329.

46. Yi, Z.; Yuan, L.; Sun, D.; Li, Z.; Wu, C.; Yang, W.; Wen, Y.; Shan, B.; Huang, Y., High-Performance Lithium-Selenium Batteries Promoted by Heteroatom-Doped Microporous 
Carbon. J. Mater. Chem. A 2015, 3, 3059-3065.

47. Chaudhari, K. N.; Song, M. Y.; Yu, J.-S., Transforming Hair into Heteroatom-Doped Carbon with High Surface Area. Small 2014, 10, 2625-2636.

48. Zhang, K.; Zhao, Q.; Tao, Z.; Chen, J., Composite of Sulfur Impregnated in Porous Hollow Carbon Spheres as the Cathode of Li-S Batteries with High Performance. Nano Res. 2012, 6, 38-46.

49. Yuan, G.; Wang, G.; Wang, H.; Bai, J., A Novel Three-Dimensional Sulfur/Graphene/Carbon Nanotube Composite Prepared by a Hydrothermal Co-Assembling Route as Binder-Free Cathode for Lithium-Sulfur Batteries. J. Nanopart. Res. 2015, 17, 1-11.

50. Xu, H.; Deng, Y.; Shi, Z.; Qian, Y.; Meng, Y.; Chen, G., Graphene-Encapsulated Sulfur (GES) Composites with a Core-Shell Structure as Superior Cathode Materials for LithiumSulfur Batteries. J. Mater. Chem. A 2013, 1, 15142-15149.

51. Zheng, G.; Yang, Y.; Cha, J. J.; Hong, S. S.; Cui, Y., Hollow Carbon NanofiberEncapsulated Sulfur Cathodes for High Specific Capacity Rechargeable Lithium Batteries. Nano Lett. 2011, 11, 4462-4467.

52. Yang, X.; Zhang, L.; Zhang, F.; Huang, Y.; Chen, Y., Sulfur-Infiltrated GrapheneBased Layered Porous Carbon Cathodes for High-Performance Lithium-Sulfur Batteries. ACS NANO 2014, 8, 5208-5215.

53. Wang, C.; Su, K.; Wan, W.; Guo, H.; Zhou, H.; Chen, J.; Zhang, X.; Huang, Y., High Sulfur Loading Composite Wrapped by 3D Nitrogen-Doped Graphene as a Cathode Material for Lithium-Sulfur Batteries. J. Mater. Chem. A 2014, 2, 5018 -5023.

54. Li, G.; Ling, M.; Ye, Y.; Li, Z.; Guo, J.; Yao, Y.; Zhu, J.; Lin, Z.; Zhang, S., Acacia Senegal-Inspired Bifunctional Binder for Longevity of Lithium-Sulfur Batteries. Adv. Energy Mater. 2015, 5, 1500878-1500885.

55. Liu, Y.; Guo, J.; Zhang, J.; Su, Q.; Du, G., Graphene-Wrapped Sulfur Nanospheres with Ultra-High Sulfur Loading for High Energy Density Lithium-Sulfur Batteries. Appl. Surf. Sci. 2015, 324, 399-404.

56. Zhou, G. M.; Yin, L. C.; Wang, D. W.; Li, L.; Pei, S. F.; Gentle, I. R.; Li, F.; Cheng, H. M., Fibrous Hybrid of Graphene and Sulfur Nanocrystals for High-Performance LithiumSulfur Batteries. ACS NANO 2013, 7, 5367-5375.

57. Ding, B.; Yuan, C.; Shen, L.; Xu, G.; Nie, P.; Zhang, X., Encapsulating Sulfur into Hierarchically Ordered Porous Carbon as a High-Performance Cathode for Lithium-Sulfur Batteries. Chem. Eur. J. 2013, 19, 1013-1019.

58. Jiang, S.; Zhang, Z.; Lai, Y.; Qu, Y.; Wang, X.; Li, J., Selenium Encapsulated into 3D Interconnected Hierarchical Porous Carbon Aerogels for Lithium-Selenium Batteries with High Rate Performance and Cycling Stability. J. Power Sources 2014, 267, 394-404.

59. Li, S.; Qiu, J.; Lai, C.; Ling, M.; Zhao, H.; Zhang, S., Surface Capacitive Contributions: Towards High Rate Anode Materials for Sodium Ion Batteries. Nano Energy 2015, 12, 224-230.

60. Li, S.; Xue, P.; Lai, C.; Qiu, J.; Ling, M.; Zhang, S., Pseudocapacitance of Amorphous $\mathrm{TiO}_{2} @$ Nitrogen Doped Graphene Composite for High Rate Lithium Storage. Electrochim. Acta 2015, 180, 112-119. 
Table of Content:

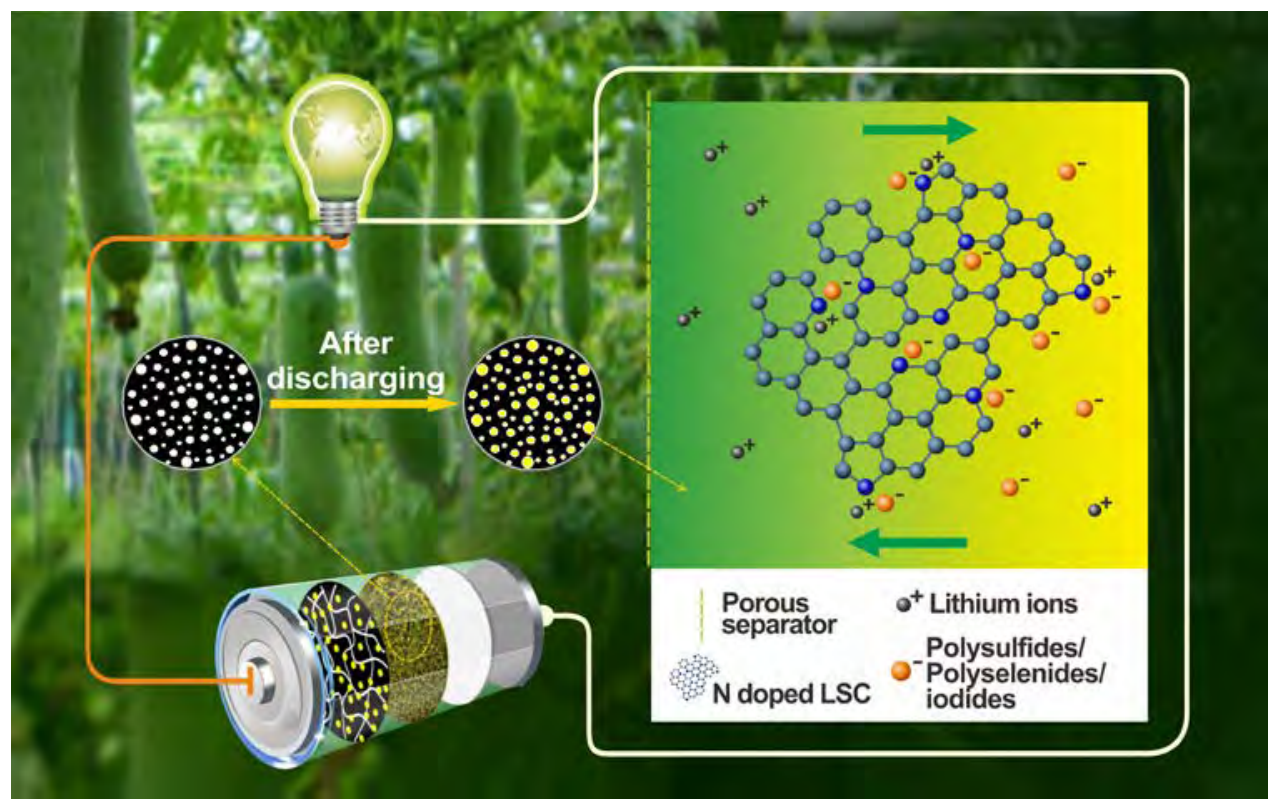

